\title{
De la familia sistémica a la familia global: Apuntes sobre la familia y sus dinámicas desde el enfoque sistémico y la sociología de la globalización
}

\section{From the systemic to the integral family: notes on the family and its dynamics from the systemic approach and the sociology of globalization}

\section{Resumen}

El concepto de familia, comprendida a la luz de la teoría sistémica, debe ser revisado, puesto que se vislumbran cambios en la realidad familiar, producto de la influencia del contexto de la globalización y el neoliberalismo. Ello implica sondear de nuevo las relaciones familiares configuradas por los procesos de migración transnacional y la virtualización de las relaciones sociales, fomentadas por las nuevas tecnologías de la comunicación y la información. Por tanto, esta revisión intenta contrastar, conceptualmente, las referencias sobre la familia y sus prácticas, teniendo en cuenta el enfoque sistémico y las investigaciones desarrolladas por la sociología de la globalización.

Palabras claves: familia, dinámicas familiares, teoría sistémica, sociología de la globalización, migración, transnacionalismo, localismo, transformación y cambios familiares.

\section{Abstract}

The concept of family, under the light of systemic theory, must be revised given that changes in the family reality are seen as a result of the influence of the context of globalization and neoliberalism, which implies revising again, the family relationships shaped by the processes of transnational migration and the digitalization of social relations, fostered by the new communication and information technologies. Therefore, this review attempts to contrast, conceptually, the references about the family and its practices, taking into account the systemic approach and the research developed by the sociology of globalization.

Keywords: family, family dynamics, systemic theory, sociology of globalization, migration, transnationalism, localism, transformation and family change. 


\section{Introducción}

Este texto aborda un contraste conceptual y teórico sobre las concepciones familiares y las acciones desarrolladas dentro de ella denominadas "dinámicas familiares". Esta comparación se realizó a partir del análisis de documentos de tipo investigativo sobre la familia desde el punto de vista de la teoría sistémica, en contraste con las generadas por los estudios sociológicos de la globalización y el transnacionalismo.

Dentro de los hallazgos encontrados, se arguye que el actual panorama de investigaciones sobre la familia y sus dinámicas está situado en la comprensión de los aspectos internos y sus relaciones locales con otros grupos humanos. Sin embargo, debido a los cambios que se han generado al interior de las familias, producto del contexto social, cultural y político, afianzados por los procesos de globalización de los mercados, conocido como neoliberalismo; se han propuesto nuevos intentos de reconceptualizar a la familia a partir de las transformaciones suscitadas por los procesos migratorios transnacionales, y la virtualización de la vida diaria producto de la inserción de las tecnologías de la información.

En consecuencia, los estudios globales sugieren que los procesos de movilidad humana y la creación de nuevas herramientas informativas que conectan diferentes realidades a escala mundial, han incidido en la desconfiguración de la familia tradicional, unificada y local; por otras formas de relación familiar denominadas "familias globales" o"familias transnacionales", cuyo surgimiento se debe a que los vínculos afectivos de tipo conyugal, fraternal y parental se desarrollan desde la distancia.

En este sentido, este tipo de familias manifiestan, dentro de sí, nuevas concepciones y prácticas sobre la paternidad y la maternidad; cambios en las concepciones sobre lo femenino y lo masculino; la transformación de las dinámicas de aprovisionamiento económico, de reproducción social, y la ampliación de redes o cadenas familiares que traspasan las fronteras nacionales; denotando su multilocalidad o su presencia en diversos sitios, sin que esto signifique que haya una fragmentación de las relaciones familiares.

En últimas, la discusión del concepto de familia y su intersección con las dinámicas socioculturales actuales, dio como resultado la visibilización de sugerencias paradigmáticas para problematizar y abordar, de manera amplia y crítica; las nuevas realidades de la familia contemporánea. En este caso, se sugiere profundizar los estudios familiares teniendo en cuenta las influencias generadas por las desigualdades sociales, económicas y políticas; así como la de estudiar las realidades familiares realizando conexiones entre lo local y lo global.

\section{La familia como sistema}

Aunque las concepciones de la familia pueden estar relacionadas con las experiencias cotidianas sobre el ser hijo, padre o madre, desde un punto de vista académico, dicha significación se ha abordado desde diversos enfoques, entre ellos, la perspectiva sistémica.

Inicialmente, como antecedente de una concepción sistémica de la familia, Urie Bronfenbrenner (1986) plantea que la familia puede considerarse un contexto o "entorno" en el que da lugar el desarrollo humano (Bronfenbrenner, 1986, p. 723), es decir, la familia se concibe como un "entorno sistémico", cuyo fin es el de posibilitar al individuo su desarrollo pleno desde su concepción (Espinal, Gimeno y González, s.f).

En este caso, la familia se le consideraría un "ambiente", o como lo podría calificar Bronfenbrener (1987), un "microsistema", donde el individuo se sitúa en un contexto inmediato en el que experimenta, percibe y siente de manera directa los acontecimientos generados por relaciones interpersonales en el que se involucra; a partir de los roles o papeles que asume y las actividades que desarrolla dentro de una cotidianidad (Bronfenbrener, 1987, p. 41).

Sin embargo, el individuo no necesariamente se desarrolla por la influencia de las relaciones que se generan dentro de este microsistema, sino que, de acuerdo con Bronfenbrenner (1986), se va desarrollando en diversos ambientes en los que se relaciona durante su recorrido vital.

Esto quiere decir que, las personas no solo emergen dentro de los ambientes familiares, tambien estos se vinculan con otros entornos o sistemas llamados a impulsar el desarrollo humano, como por ejemplo, 
las influencias generadas por las relaciones sociales con miembros de su comunidad inmediata (mesosistema) o las relaciones indirectas con las instituciones sociales o políticas de otra índole (exosistemas), y la influencia de los aspectos culturales e ideológicos dentro de las concepciones que posibilitan o imposibilitan el desarrollo humano (macrosistema) (Bronfenbrener, 1987, p. 41).

De acuerdo con lo anterior, el desarrollo del individuo se sitúa en diversos niveles contextuales, considerando a la familia como un punto de inicio debido a que es referenciada como parte de un "ambiente ecológico" (Bronfenbrener, 1987) más amplio. En otras palabras, la familia sería conceptualizada como el ambiente natural donde emergen los individuos.

Siguiendo con esta línea argumental, los autores expertos en terapia familiar con enfoque sistémico, han desarrollado algunas variantes conceptuales sobre el significado de la familia. En este caso, Zea (2008) citando a Salvador Minuchin (1998) plantea que el concepto de la familia puede ser entendida como

(...) un grupo de personas, unidas emocionalmente y/o por lazos de sangre, que han vivido juntos el tiempo suficiente como para haber desarrollado patrones de interacción e historias que justifican y explican tales patrones. En sus interacciones modeladas con el otro, los miembros de la familia se construyen entre sí (Zea, citando a Minuchin, p. 56).

A diferencia de Bronfenbrenner, para Minuchin (citado por Zea, 2008) la familia es catalogada como un grupo que se forja a partir de la interacción entre sus miembros, cuya identificación (papá, mamá, hijos, abuelos, tios, primos) determina la función dentro de dicha organización social.

Por otro lado, debido a que la interacción es casi constante entre los miembros de la familia, se pueden dar relaciones de negociación recíproca que permiten una reorganización de los valores, representaciones familiares y acciones, conllevando que la familia se dinamice a partir de los eventos o sucesos cotidianos que se suscitan en el compartir de las experiencias dentro del ámbito privado. Dicha interacción estaría modelando concepciones de pertenencia grupal y conocimientos sobre los comportamientos pro- pios de los miembros de la familia ante determinadas situaciones.

Así, para Minuchin, Lee y Simon (1998) la familia se comprende desde dos perspectivas: la configuración familiar y la evolución familiar. La primera hace referencia a la composición demográfica familiar, es decir, si se trata de una familia nuclear, extensa, monoparental, ensamblada u homoparental. Por otro lado, el concepto de evolución familiar se refiere a las etapas del ciclo vital y a los ajustes que las familias deben ejercer dependiendo de la etapa en la cual se encuentren; esta perspectiva considera los cambios contextuales que en cada etapa se producen (Minuchin, Lee y Simon, 1998).

Teniendo en cuenta estos aspectos, el ser humano es influido por el contexto social, y este a su vez influye sobre su contexto. Por eso, para Minuchin (2001) la familia también constituye un contexto para un individuo, es un grupo social natural, dado que las respuestas de sus integrantes están determinadas por la interacción con otros sistemas sociales externos a la familia, al igual que con la interacción que se desarrolla al interior de la familia, es decir, en el ámbito privado.

Así, los miembros de la familia generan relaciones de interdependencia, donde el individuo es influido por su contexto y este influye sobre lo social. La vida psíquica de un individuo no obedece exclusivamente a aspectos internos, sino también a situaciones socioculturales, medioambientales y políticas, implicando con ello que el individuo debe adaptarse a un sistema particular, en este caso, al sistema familiar.

Por otro lado, Carlos Sluzky a partir de su concepto de sistemas de redes, manifiesta que la familia sería una "red social personal" (Sluzky, 1996, p. 42) para el individuo. Es decir, la familia sería un entorno generado por relaciones íntimas cotidianas que se desarrollan en privado, conformando así una red social que se va ampliando con redes externas a la familia, a partir de la generación o invincración de relaciones entre sí, ya sea con redes comunitarias, redes laborales o redes institucionales de tipo político y culturales (Sluzky, 1996).

En consecuencia, dentro de la concepción sistémica, se plantean diversos modos de entender a la familia. En este caso, Hernandez (1997) sintetiza que la familia 
puede concebirse como un sistema institucional, grupal, cultural o como un sistema de relaciones afectivas.

En cuanto a la familia como sistema de institución social, esta noción implica comprender que los miembros están regulados por normas y reglas de comportamiento que garantizan ciertas funciones familiares, como, por ejemplo, el adecuado funcionamiento biológico de los individuos de la sociedad, la reproducción de sus miembros, su adecuada socialización, la provisión y distribución de los bienes y servicios, el mantenimiento del orden dentro del grupo familiar de acuerdo a los preceptos sociales generales, la definición del sentido de vida y la motivación para preservar la supervivencia individual y grupal (Collins, 1987, citado por Hernández, 1997).

En cuanto a la familia significada como un grupo, para Hernández (1997), las interacciones cotidianas de sus miembros contribuyen a preservar la supervivencia de los individuos en familia, convirtiéndose tanto en receptora del contexto como en causante de cambios contextuales. Por otro lado, la familia conceptualizada como construcción cultural, es concebida en que las acciones de sus miembros reflejan los valores sociales, tradicionales, religiosos y políticos de su entorno y en las formas de relación que son establecidas por la cultura, pero que pueden modificarse de acuerdo a las particularidades de cada familia.

Por último, la noción de la familia teorizada como un conjunto de relaciones emocionales se concibe como una entidad que se establece para satisfacer las necesidades afectivas de sus miembros a través de sus dinámicas familiares, que pueden a su vez, facilitar, o por el contrario, dificultar la satisfacción de tales necesidades (Hernández, 1997, p.14).

Por lo tanto, para Hernández (2005) la familia se concebiría como:

(...) una unidad ecosistémica, que crea solidaridades de destino en el tiempo y en el espacio y opera a través de rituales, mitos y epistemes, que se organizan en el interjuego de procesos filogenéticos, ontogenéticos y culturogenéticos" (p. 6).

Es decir, que la familia es concebida como un sistema interaccional que responde a necesidades biológicas, psicológicas y culturales indispensables para la su- pervivencia tanto del individuo, como de la sociedad, puesto que en ella se construye una red de relaciones sociales influidas por conocimientos y prácticas culturales que se imbrican para generar espacios particulares de interacción dentro de un tiempo concreto. En otras palabras, cada familia manifiesta unas características particulares que llevan a cabo para garantizar la supervivencia y la satisfacción de necesidades psicoafectivas de sus miembros, en un espacio y en un periodo histórico determinado.

De acuerdo con esta postura, la unidad de análisis son las interacciones o relaciones que mantiene los sujetos, siguiendo patrones regulares o irregulares que permite verificar cambios dentro de los contextos familiares. Concretamente, se intentan verificar los modos de interacción a partir de la composición, jerarquía, límites y roles dentro de los sistemas familiares; las funciones de los miembros, la variabilidad de las normas y de los significados en que operan dichos reglamentos, y el desarrollo evolutivo familiar, a partir de su flexibilidad y capacidad para adaptarse a los cambios internos y externos demarcados por el ciclo vital o por eventos del entorno que exigen modificaciones en los patrones de interacción (Hernandez, 1997, p. 28).

En síntesis, comprender a la familia como sistema tiene que ver con estudiar las relaciones e interacciones en los que se lleva a cabo el desarrollo de los individuos, a partir de sus comportamientos y percepciones sobre los hechos que les afectan en un tiempo presente y en un lugar concreto, es decir, el sistema familiar sería una metáfora que implica aprender su significado como un "ambiente grupal social" que se genera a partir de las relaciones entre las personas. Este nuevo ambiente cimenta una estructura interaccional que inicia desde una posición asignada por papeles o roles que funcionan de manera dinámica entre sus miembros y que suelen cambiar, producto de las influencias generadas por las relaciones con otros entornos de tipo cultural, político, comunitario y ambiental.

Si bien es cierto que los preceptos de la teoría sistémica explica a la familia como un grupo natural formado por relaciones interpersonales que estructuran y funcionan como un subsistema en medio de sistemas más generales, ha implicado que dicha concepción observe solo los papeles, funciones y estructuras que cada individuo manifiesta en sus núcleos fami- 
liares y en las relaciones que se generan por fuera de ella, dentro de una localidad. Es decir, que estos grupos humanos son entidades sociales fijas que se asientan dentro de un determinado espacio territorial y temporal, cuya dinámica únicamente se va a generar en relación con otros sistemas sociales que están situados en su periferia. Por lo tanto, hay una visión localista, topográfica, geográfica y situada en un tiempo presente de la realidad familiar.

Asimismo, al parecer es poco lo que se tiene en cuenta los aspectos dinámicos y cambiantes de los procesos identitarios de tipo social y subjetivo que se van configurando y transformando producto de procesos culturales, históricos, económicos y sociales generados por desigualdades, luchas identitarias y situaciones políticas de tipo local y global.

Por consiguiente, es necesario comprender a la familia no solamente como un ambiente o un espacio concreto que se genera por relaciones sociales directas e indirectas, sino que es primordial también pensarla a partir de las transformaciones que se han venido generando a la luz de las nuevas realidades que la afectan en el presente. Para este caso, hablamos del contexto de la globalización y el libre mercado propiciado por el neoliberalismo económico, las nuevas tecnologías de la información, y la migración transnacional.

\section{La familia global}

La concepción de una familia naturalizada, estática, y tradicional, se ha venido transformando producto de las nuevas relaciones sociales actuales basadas en la virtualidad, la fragilidad de los vínculos sociales, y la profundización de la individualización generada por la globalización económica y tecnológica (Bauman, 2006); así como también por la influencia de los procesos de migración y las relaciones laborales transnacionales (Beck y Beck-Gernsheim, 2012).

Por tales motivos, las relaciones sociales actuales han impulsado a que los papeles, roles, estructuras y funciones familiares se manifiesten de manera difusa y poco claras, es decir, que el concepto de familia como grupo o contexto estable cambia debido a que las relaciones contemporáneas de la vida privada son momentáneas y susceptibles de transformación lo que significa que el grupo familiar en la actualidad varía constantemente (Bauman, 2006).

Teniendo en cuenta lo anterior, estas transformaciones han traído consigo cambios en diversas esferas de la vida familiar. Entre ellas se destacan la vida laboral de las figuras parentales (vinculación de la mujer a la vida laboral) (Martínez Quintana, 2010), cambios de pareja sentimental de manera constante (separaciones, divorcios), el arraigo de los métodos de anticoncepción y control natal (Cetavorev, 2003), la vinculación de nuevas identidades de género en la conformación de nuevas familias que rivalizan con la familia heterosexual, el acceso a derechos igualitarios (mujeres y grupos LGTBI) (Quintero, 2009); y las familias que se configuran alrededor del transnacionalismo (Bricesón y Vourella, 2002; Sorense, 2008; Escobar, 2008; Cerda, 2014).

En efecto, ante las situaciones mencionadas, los sociólogos Beck y Beck-Gernsheim (2012) plantean la existencia de nuevas realidades familiares emergentes, denominadas familias globales, las cuales son conceptualizadas como:

(...) relaciones amorosas y de parentesco entre personas que viven en distintos países o continentes, o de diferentes procedencias de países y continentes y que pueden adoptar una gran diversidad de formas y fundamentarse en una gran variedad de motivos y razones. (...) las familias globales representan una novedosa mezcla de cercanía y distancia, de igualdad y desigualdad, y de seguridad e inseguridad (Plumed, 2013, p. 93, citado por Beck y Beck-Gernsheim, 2012).

Dicho lo anterior, el panorama social que ha implicado el contexto de la globalización, ha conllevado que los núcleos familiares se transformen de acuerdo a los cambios económicos, políticos y tecnológicos de las sociedades contemporáneas.

En este caso, Oliva yVilla (2014) manifiestan que la familia dentro del contexto de la postmodernidad y la globalización

(...) está atravesada no solo por los efectos socioculturales inmediatos cuando se interactúa con otros grupos sociales; también se debe tener en cuenta la influencia de los medios tecnológicos informativos en el surgimiento de nuevos lazos familiares y relaciones afectivas a partir de la ampliación de los marcos comunicativos 
que posibilitan conexiones a larga distancia, conllevando con ello la construcción de nuevas parejas o familias que surgen de la interacción mediática. Para ser más específicos, las herramientas comunicativas virtuales tales como el internet, ya hacen parte como elementos notorios de configuración de nuevas formas familiares (p. 18).

No obstante, no solo el surgimiento de las nuevas tecnologías de la información ha tenido un papel importante en la configuración de la nueva realidad familiar en el contexto de la globalización. Lo que al parecer ha generado dichos cambios y transformaciones familiares actuales, son las movilidades humanas, es decir, los procesos de migración y las diásporas de tipo transnacional.

\section{La familia transnacional}

Los procesos migratorios y las diásporas tienen que ver con la movilidad de personas a regiones y naciones diferentes a su lugar de origen, llevando tras de sí sus formas de vida familiar, cultural y social al pasar las fronteras establecidas por los Estados - Nación.

En este caso, los estudios migratorios y transnacionales señalan las influencias, los efectos y consecuencias que las movilidades humanas generan en la transformación de la vida familiar. Así, Bricesón y Vourella (2002; citados por Escobar, 2008) plantean el término "familia transnacional" el cual puede referirse a

(...) aquella familia que vive buena parte o la mayor parte del tiempo separada. Sin embargo, a pesar de la distancia, esta logra mantenerse unida dando paso a un sentimiento colectivo de bienestar y de unidad que supera las fronteras nacionales (p. 247).

En efecto, la familia se puede conceptualizar como una entidad social "multilocal" o "multisituada" (Gonzalvez, 2007) debido a que las relaciones de tipo afectivo (conyugal y fraternal), los papeles sobre la paternidad, la maternidad, la niñez, y la asignación de funciones tales como los cuidados familiares, la regulación social de los miembros, la vida sexual y laboral de las parejas, se van transformando y construyendo en múltiples contextos nacionales y culturales producto de los intercambios con identidades, costumbres, normas y reglas foráneas, que hacen dinamizar las funciones, estructuras, papeles y la manera en cómo se resuelven los conflictos en la familia cuando uno de sus miem- bros se encuentra fuera de ella (Escobar, 2008).

Así pues, las familias transnacionales tienen que ver con establecer conexiones, eslabones o cadenas de tipo emocional-asistencial (Russell, 2001) que permiten mantener a la familia como organización primordial de socialización, aunque lo particular de estas familias es que se van transformando y, a la vez, emergen conflictos constantemente, a medida que sus miembros diversifican sus movilidades migratorias.

Precisamente, algunos aspectos que definen a las familias como transnacionales están relacionados, en primera medida, con el modo de vinculación emocional que llevan a cabo en la distancia y se mantiene a través de"sentimientos de lealtad y por proyecciones de un hogar imaginado generados entre sus miembros" (Parella, 2012, p. 663).

Por otro lado, se les define también por sus prácticas o mecanismos para reforzar sus vínculos afectivos transnacionales, tales como las remesas monetarias, la comunicación constante que posibilita las nuevas tecnologías de la información (TIC) entre parientes (Escobar, 2008), y la utilización de redes familiares para ejercer los cuidados de los hijos mientras los padres y madres migrantes están ausentes (Zapata, 2009).

En este caso, las familias crean estrategias de preservación de vínculos familiares a través de las fronteras, ya que estos mecanismos permiten a los miembros migrantes sortear las dificultades familiares, y así cumplir, tanto con las obligaciones parentales, como las conyugales (Zapata, 2009).

Sin embargo, más allá de estas características, Parella (2012) señala que las familias transnacionales están atravesadas por procesos más complejos de tipo estructural que

obedecen al surgimiento de desigualdades sociales dentro de ellas, ya sea por políticas migratorias que condicionan a los sujetos transnacionales a tener o no un reconocimiento legal de su ciudadanía (legales e ilegales); los problemas económicos y condiciones laborales que motivan a los miembros de estas familias modificar sus rutas y destinos constantemente, la modificación de papeles y roles en el desempeño en el hogar producto de la renegociación y transformación de las relaciones de poder, tales como las relaciones de género (hombre - mujer) y relaciones entre generaciones(padres -hijos-familiares), el bienestar de sus miembros y la construcción de nuevas identidades (p. 664). 
Sintetizando estos aspectos, Cerda (2014) manifiesta que las familias transnacionales se pueden caracterizar por diversos motivos, entre los cuales están: la desterritorialización de las relaciones familiares; la conyugalidad se define a distancia; la paternidad y la maternidad es de manera semipresencial, es decir, la ausencia y presencia de autoridades familiares; la provisión económica y la reproducción social de la familia se basa en el envío de remesas monetarias; y se incluyen nuevos miembros a la familia que no están ligados a vínculos consanguíneos.

Por último, son familias que se encuentran en mayor riesgo de vulnerabilidad social producto de las diversas condiciones y problemáticas que cada país o territorio nacional presenta en el ámbito político, social, económico y cultural. Lo anterior se debe a que las familias transnacionales sortean las restricciones impuestas por políticas migratorias y de ciudadanía; así como los dilemas generados por la xenofobia y la precarización laboral; llevando a que los miembros de dichas familias se movilicen constantemente de un territorio nacional a otro, y su adaptación social no sea tan regular (Cerda, 2014).

Así pues, vemos que el surgimiento de esta clase de familia deviene de situaciones migratorias producto de condiciones sociopolíticas y culturales que se están presentando en la actualidad. En este caso, familias que debido a la situación de desigualdad económica y social presentes en sus lugares de origen, se configuran en la distancia debido al éxodo de sus miembros, o personas migrantes que se convierten miembros de una familia producto de una relación contractual de servicios asistenciales de cuidados familiares (niñera, servicio doméstico); o familias que se configuran producto de relaciones sentimentales transnacionales y que se sostienen mediante las nuevas formas de comunicación -internet-; o por el contrario, personas que se convierten en madres"de alquiler" que son contratadas por personas que no pueden concebir un nuevo miembro familiar (Beck y Beck-Gernsheim, 2012; Plumed, 2013).

En consecuencia, la realidad de la familia de hoy día se está modificando a luz de los procesos políticos, sociales, económicos y culturales, conllevando a que nuevas configuraciones sociales surjan, tal como la familia global o transnacional.
Finalmente, las familias desde una perspectiva global, tienden a configurarse en relaciones momentáneas, es decir, no se mantienen en el tiempo. Los roles y papeles de los miembros de la familia varían de acuerdo a las concepciones que se le otorgan sobre la vida parental y a las identidades que se van construyendo en las relaciones público-privadas. Estas nuevas familias son dinámicas, es decir, la familia contemporánea se moviliza producto de los procesos de migración y el transnacionalismo laboral, donde la cohesión familiar no necesariamente se genera alrededor de la cercanía de sus miembros, sino a través de relaciones a larga distancia mantenidas por relaciones virtuales.

\section{Apuntes sobre las dinámicas familiares}

Si tenemos en cuenta las diversas conceptualizaciones sobre la familia, las dinámicas familiares también pueden abarcar diversas definiciones teniendo en cuenta los marcos teóricos que van sustentado las compresiones sobre las relaciones y modos de convivencia entre los miembros que conforman una familia. Es este caso, desde un enfoque sistémico, Agudelo (2005) plantea que las dinámicas familiares consisten en:

(...) las diversas situaciones de naturaleza psicológica, biológica y social que están presentes en las relaciones que se dan entre los miembros que conforman la familia y que les posibilita el ejercicio de la cotidianidad en todo lo relacionado con la comunicación, afectividad, autoridad y crianza de los miembros y subsistemas de la familia, los cuales se requieren para alcanzar el objetivo fundamental de este grupo básico de la sociedad: lograr el crecimiento de los hijos y permitir la continuidad de las familias en una sociedad que está en constante transformación (p. 9).

De acuerdo con esta perspectiva, las dinámicas se describen desde un punto de vista interno. En otras palabras, las interacciones entre los miembros que conforman una familia se basan tanto en cuestiones individuales (motivaciones, creencias, sentimiento, razonamientos), biológicas (reproducción, alimentación, crecimiento físico, salud) y sociales (políticas, culturales) en las que la crianza, la comunicación, la afectividad y la autoridad se sustentan como prácti- 
cas cotidianas para asumir retos, dificultades, éxitos y fracasos (Gallego, 2011).

Así, una interpretación sobre esta definición la realiza Gallego (2011):

(...) la dinámica familiar se puede interpretar como los encuentros entre las subjetividades, encuentros mediados por una serie de normas, reglas, límites, jerarquías y roles, entre otros, que regulan la convivencia y permite que el funcionamiento de la vida familiar se desarrolle armónicamente. Para ello, es indispensable que cada integrante de la familia conozca e interiorice su rol dentro del núcleo familiar, lo que facilita en gran medida su adaptación a la dinámica interna de su grupo (p. 333).

De acuerdo con estas afirmaciones, se infiere que las dinámicas familiares son relaciones funcionales que se presentan al interior de las familias como un modo de adaptabilidad social que permite el normal funcionamiento de las relaciones hogareñas.

Sin embargo, de acuerdo con Torres, Ortega, Garrido y Reyes (2008), concebir las dinámicas familiares como el estudio de relaciones armoniosas a partir de supuestos como la unidad y el interés común dentro de los miembros de un hogar, desdibuja la realidad sobre las diferencias, desigualdades y conflictos que se presentan al interior de las familias. Por lo tanto, para estas autoras, las dinámicas familiares pueden ser concebidas como:

(...) el conjunto de relaciones de cooperación, intercambio, poder y conflicto que, tanto entre hombres como mujeres, y entre generaciones, se establecen en el interior de las familias, alrededor de la división del trabajo y de los procesos de toma de decisiones (Torres, et. al., 2008, p. 33).

Lo anterior implica que el estudio de las dinámicas familiares no solo aborda el modo interno en que se desenvuelven los miembros de una familia cuando se relacionan entre sí (roles, comunicación, crianza, autoridad), sino que dichas acciones se ven influenciadas por significaciones y prácticas sociales externas a la familia que pueden definir desigualdades sociales y conflictividades a partir de concepciones relacionadas con el papel de la mujer y el hombre en el hogar, el trabajo, la crianza, la manutención económica, la educación, los intereses individuales, la mediación y resolución de los conflictos, las relaciones jerarquizadas y de poder entre las parejas y hacia los hijos, entre hermanos, los ideales sobre el futuro individual y los aspectos ideológicos sustentados en la política y la religión.

Todo lo anterior son elementos claves que dinamizan y complejizan la vida familiar y le dan un tinte particular sobre las vivencias que cada hogar expresa en su cotidianidad, teniendo en cuenta que dichas vivencias privadas están en conexión constante con los aspectos cotidianos de la esfera pública.

\section{Dinámicas familiares, globalización y transnacionalismo}

Desde la perspectiva del transnacionalismo y la sociología de la globalización, las dinámicas familiares se van a conectar con factores relacionados con los flujos migratorios transnacionales, las influencias de la informaciones y contenidos que circulan en el mundo virtual y digital, los vaivenes de la economía global y sus repercusiones en la reproducción de nuevas desigualdades sociales, como también los cambios y luchas políticas situadas en la defensa de identidades colectivas e individuales manifestadas en el reconocimiento de sujetos políticos basados en el género, la etnia y en la clase social.

En este caso, la virtualidad y digitalidad de la comunicación familiar, el aprovisionamiento de recursos monetarios y materiales a partir de las remesas económicas, la fragilidad de las relaciones conyugales, la flexibilidad de los papeles parentales, maternales y filiales; las prácticas de cuidado, de afecto y asistencial de tipo contractual o solidario a partir de redes familiares (Russell, 2001; Merla 2014), y el manejo de los conflictos entre padres e hijos con ausencia de alguno o de los dos progenitores, develan lo que Quilodran y Castro (2009) señalan como las"nuevas dinámicas familiares" nacidas producto de la influencia que ejerce el transnacionalismo y los procesos de globalización en la transformación de la vida familiar contemporánea. De acuerdo con Pedone (2011), dichas transformaciones de las dinámicas familiares en el contexto del transnacionalismo, están relacionadas con el 
(...) reacomodo de la estructura y dinámica de los grupos domésticos, los patrones de conyugalidad, las prácticas de crianza, y los procesos de independencia e individuación de hijos e hijas, cuya transversalidad está dada por los ajustes y reelaboraciones de las relaciones de género y generacionales fomentadas por los procesos de migración (p. 231).

Por una parte, los efectos del transnacionalismo generaron en las dinámicas familiares el reacomodo de las prácticas de cuidado y asistencia, en el que su transformación ha establecido, por un lado, redes y círculos solidarios entre grupos familiares que apoyan y redefinen los vínculos familiares cuando los sujetos proveedores de cuidados y de afecto a personas dependientes (hijos, hijas, personas mayores de edad) se encuentran ausentes por la migración, configurándose una "circulación de cuidados" (Merla, 2014, p. 88), es decir, una red de personas que asumen papeles de cuidado pertenecientes o allegadas a una familia. Estas personas desempeñan los cuidados y asistencia de manera solidaria y no necesariamente son contratadas o remuneradas (Merla, 2014, citando a Barldassar y Merla, 2014; Krmpotic y De Ieso, 2010).

Por otro parte, se han configurado cuidados de atención y asistencia de tipo remunerado y contractual en las que las personas migrantes dejan de lado los cuidados de sus familias de origen para realizar prácticas de maternidad y de asistencia en otras familias, y estas, a su vez, contratan un tercero para que se haga cargo de la responsabilidad de los cuidados dentro de sus familias. Dicha dinámica configura lo que Russell (2001) denomina"las cadenas mundiales de afecto o de asistencia" (Russell, 2001, p. 188), es decir, un entramado de trabajos relacionados con la crianza, cuidados y asistencia a nivel global, realizados en su mayoría por mujeres migrantes.

Sin embargo, como lo señala Merla (2014), las personas migrantes que dejan a cargo las prácticas de cuidado y asistencia a terceros, siguen desarrollando estas acciones que le corresponden en sus familias, pero a distancia. En este caso, los cuidados personales,

(...) el apoyo financiero y material a partir del envío de remesas de dinero y objetos; el apoyo emocional, el manejo de la autoridad, la consejería, la asistencia de actividades de la vida cotidiana; y la provisión de alojamiento se realizan por parte de padres o madres migrantes a partir de la utilización de los medios de comunicación virtuales tales como el internet, videollamadas, salas de chats, o medios de comunicación digital como la telefonía móvil, fax, etc. (Merla, 2014, p. 90, citando a Baldassar, 2007).

Si bien es cierto que la comunicación familiar, en el contexto de la globalización y el transnacionalismo, ha sufrido una transformación debido a que las relaciones comunicativas familiares se desarrollan de manera virtual y a distancia, lo cierto es que las herramientas tecnológicas digitales han jugado un papel importante en el mantenimiento de los núcleos familiares.

Como lo señala Solé y Parella (2006), el papel de las Tecnologías de la Información (TIC) en la comunicación de las familias transnacionales es de gran importancia, ya que permite afianzar las relaciones sentimentales y de lealtad, tomar decisiones en familia y gestionar los recursos y procesos de cuidados y de crianza, al igual que suavizar los dilemas emocionales generados por la distancia a nivel conyugal como intergeneracional. Todo esto debido a que:

(...) los servicios virtuales o digitales como los café-internet y los celulares generan una comunicación en tiempo real, lo que hace que las familias transnacionales puedan mantenerse unidas a partir de la creación de nuevos espacios de comunicación familiar facilitados por este tipo de herramientas (p. 8).

Por último, en conexión con lo anterior, las prácticas de remesas monetarias y materiales que consisten en el envío de dinero y objetos por parte de padres y madres migrantes para el mantenimiento de la familia, es un aspecto importante el cual se podría atribuir como una forma de nueva dinámica familiar, debido a que las remesas afianzan la responsabilidad de los padres y madres migrantes de atender las necesidades y el bienestar de sus hijos u otros miembros, reforzando así los lazos afectivos conyugales e intergeneracionales en estas familias (Zapata, 2009).

En síntesis, las dinámicas familiares transnacionales están basadas en poder afianzar los lazos de solidaridad y mantener vínculos emocionales y lealtades entre sus miembros. Igualmente, sortear las dificultades y conflictos generados por la ausencia de padres y madres que 
por circunstancias económicas, políticas o de otra índole decidieron migrar a otros países. Por lo tanto, las dinámicas familiares no solo se presentan en un ámbito interno, sino que estas prácticas se van dinamizando de acuerdo a las necesidades y procesos que se generan a la luz de las consecuencias e influencias de los aspectos socio-políticos, económicos y tecnológicos que fomentan la transformación y la emergencia de nuevas dinámicas familiares.

\section{Conclusión}

Teniendo en cuenta esta revisión documental sobre el concepto de familia y las dinámicas familiares, se concluye que las acepciones sobre la familia, desde un punto de vista sistémico, tienden a situar este grupo social en un examen dual, el cual, se evalúa, en primer lugar, si los miembros de la familia mantienen sus funciones de acuerdo a los roles asignados dentro del hogar; por otro lado, se examina si una estructura familiar dada permite un desarrollo óptimo de los sujetos.

Lo anterior tiene como consecuencia, que las evaluaciones familiares sean de tipo local, sin tener mucha atención sobre las influencias y las transformaciones que los procesos globales han generado en la redefinición de identidades, los papeles sociales y las mismas dinámicas familiares dentro de la vida privada de las familias. Esto es debido a que se concibe a la familia como algo natural, con poco espacio de movilización.

En cuanto a los estudios sobre la influencia de la globalización y el transnacionalismo en la familia, estos aplican los aspectos contextuales y socio-políticos que indican la articulación del ámbito público a los procesos privados familiares, sobre todo, se enfatizan en las transformaciones que se presentan alrededor de las relaciones sentimentales laborales y de cuidado familiar, producto de los procesos de migración transnacional, los avances tecnológicos en la comunicación, la divulgación de la información, los procesos de reconocimiento de identidades y los cambios económicos de tipo global. Asimismo, se señalan las consecuencias, dificultades y riesgos que han tomado las familias a partir de los estudios de las desigualdades sociales a partir del género, la raza y la clase social, las relaciones de poder generadas por las diferencias identitarias y de clase, como también el examen de las políticas migratorias que permean a los sujetos migrantes y profundizan las dificultades en las llamadas familias transnacionales.
Por tanto, se explica a la familia conectada con otras realidades, con otras familias, con otros grupos sociales, con situaciones adversas, con políticas y procesos de reconocimiento generados alrededor del mundo, lo que implica que la globalización permea el ámbito familiar, y como tal, genera una visión multidimensional de la familia en constante transformación.

En definitiva, se recomienda que los estudios en familia tengan en cuenta los aspectos privados, públicos y globales en el cual participan los grupos familiares ya que a partir de un análisis de estos ámbitos, se puede desarrollar un estudio no dual, sino transdisciplinar de las relaciones, transformaciones y dilemas familiares que se presentan en el contexto actual.

\section{Referencias bibliográficas}

Agudelo Bedoya, M. E. (2005). Descripción de la dinámica interna de las familias Monoparentales, simultáneas, extendidas y compuestas del municipio de Medellín, vinculadas al proyecto de prevención temprana de la agresión. Revista Latinoamericana de Ciencias Sociales, Niñez y Juventud. 3, 1, $1-19$.

Bauman, Z. (2006). Amor Líquido, acerca de la fragilidad de los vínculos humanos. Fondo de Cultura Económica. Buenos Aires.

Bronfenbrenner, U. (1986). Ecology of the Family as a Context for Human Development: Research Perspectives. Developmental Psychology. 22, 6, 723-742.

Bronfenbrenner, U. (1987). La Ecología del Desarrollo Humano. Paidós. Buenos Aires.

Cebotarev, N. (2003). Familia, socialización y nueva paternidad. Revista Latinoamericana de Ciencias Sociales, Niñez y Juventud. 1, 2,1-19.

Cerda, J. (2014). Las familias transnacionales. Revista Espacios Transnacionales. 1, 2, 78-88.

Beck, U. y Beck-Gernsheim, E. (2012). Amor a distancia. Nuevas formas de vida en la era global. Paidós, Barcelona.

Bryceson, D. yVuorela, U. (2002). The Transnational Family. New European Frontiers and Global Networks. Berg Publishers. Oxford / NewYork. 
Burgos, J.M. (2004). ¿Es la familia una institución natural? Cuadernos de Bioética. 16, 3, 359-374.

Crespo, J. M. (2011). Bases para construir una comunicación positiva en la familia. Revista de Investigación en Educación. 9, 2, 91-98.

Escobar, A. (2008). Tras las huellas de las familias migrantes del cantón Cañar. En: Herrera, G. y Ramírez, J. (Eds.). (2008). América Latina migrante: Estado, familia, identidades, 243258. FLACSO. Quito.

Espinal, I., Gimeno, A. y González, F. (s. f.). El enfoque sistémico en los estudios sobre la familia. Revista internacional de sistemas. 1, 14, 21-34. Recuperado de https://wwww.uv.es/jugar2/ Enfoque\%20Sistemico.pdf

Gallego, M. A. (2011). Recuperación crítica de los conceptos de familia, dinámica familiar y sus características. Revista Virtual Universidad Católica del Norte. 1, 35, 326-345.

Gonzálvez, H. (2007). Reseña de the transnational family. New european frontiers and global networks de Deborah Bryceson and Ulla Vuorela (Eds.) Revista de Antropología Iberoamericana. 2, 3, 584- 589 .

Hernández Córdoba, B.A. (1997). Familia, ciclo vital y psicoterapia sistémica breve. En: Colombia 1997.Vol 1., p. 192. Ed. El Búho.

Hernández Córdoba, B. A. (2005). La familia como unidad de supervivencia, de sentido y de cambio en las intervenciones psicosociales: intenciones y realidades. Revista Latinoamericana de Ciencias Sociales, Niñez y Juventud. 3, 1,57 - 71.

Krmpotic, C.S y De Ieso, L. (2010). Los cuidados familiares. Aspectos de la reproducción social a la luz de la desigualdad de género. Rev. Katál. Florianópolis. 13, 1, 95-101.

Minuchin, S., Lee, W.Y.y Simon, G. M. (1998). El arte de la terapia familiar. Paidos. Barcelona.

Martínez Quintana, V. (2010). Conciliación de la vida familiar y laboral: cambios sociales y tendencias de futuro. Revista Ábaco. 2 (49-50), 170-180.

Oliva, E. y Villa, V.J. (2014) Hacia un concepto interdisciplinario de la familia en la globalización. Justicia Juris. 10, 1, 11-20.
Parella, S. (2012). Familia transnacional y redefinición de los roles de género. El caso de la migración boliviana en España. Papers. 97, 3, 661-684.

Pedone, C. (2011). Familias en Movimiento. El abordaje teórico-metodológico del transnacionalismo familiar latinoamericano en el debate académico español. Revista latinoamericana de estudios familiares. 3, 1, 223-244.

Plumed, M. (2013). Amor a Distancia: Nuevas Formas deVida en la Era Global. [Reseña de libro]. En: International Journal of sociology of education, 93-94. 2, 1.

Quilodran, J. y Castro, T. (2009). Nuevas Dinámicas Familiares. Estudios demográficos y urbanos, 24 (2), 283-291.

Quintero, A. M. (2009). Contingencias de las Estructuras Familiares del Milenio. El Ágora Usb. 9, 2, 307-326.

Russell, A. (2001). Las cadenas mundiales de afecto y asistencia y la plusvalía emocional. En: Hutton, W.y Giddens, A. (Eds). (2001) En el Limite. La vida en el capitalismo global, 187-208. Tusquets Editores. Barcelona.

Torres, L., Ortega, S., Garrido, A. y Reyes, A. (2008). Dinámica familiar en familias con hijos e hijas. Revista Intercontinental de Psicología y Educación. 10, 2, 31-56.

Slusky, C. (1996). La red social. frontera de la práctica sistémica. Gedisa. Buenos Aires.

Solé, C. y Parella, S. (2006). El papel de las TIC'S en la configuración de las familias transnacionales"En: Sistemas, cibernetica e informática. 3, 7-12.

Sorense, N. (2008). La familia transnacional de latinoamericanos/as en Europa. En: Herrera, G. y Ramírez, J. (Eds.) (2008). América Latina migrante: Estado, familia, identidades, 259-279. FLACSO. Quito.

Zapata, A. (2009). Familia transnacional y remesas: padres y madres migrantes. En: Revista Latinoamericana de Ciencias Sociales, Niñez y Juventud. 7, 2, 1749-1769.

Zea, L. F. (2008). Comportamiento y Lenguage. Universidad EAFIT. Medellin. 